\title{
BEM-ESTAR SUBJETIVO E RESILIÊNCIA EM PESSOAS COM DIABETES MELLITUS
}

\author{
Maria da Penha de Lima Coutinho \\ Instituto de Educação Superior da Paraíba \\ Fabrycianne Gonçalves Costa \\ Instituto de Educação Superior da Paraíba \\ Márcio de Lima Coutinho \\ Instituto de Educação Superior da Paraíba
}

\begin{abstract}
Resumo
Este artigo objetivou mensurar os níveis de Bem-Estar Subjetivo (BES) e de resiliência em diabéticos, além de verificar a correlação entre a resiliência e os fatores que compõem o bem-estar subjetivo. Foram aplicadas as escalas de BES e de resiliência em 104 diabéticos (média de idade=56,16; $\mathrm{DP}=13,01$ ), residentes na cidade de João Pessoa- PB. Os resultados evidenciaram que $65,4 \%$ dos participantes apresentaram altos níveis de BES. Quanto à resiliência, constatou-se que a maioria possui entre moderada $(63,5 \%)$ e alta capacidade $(28,8 \%)$, além de melhor desempenho na dimensão de ações e valores. Observou-se também, correlação positiva entre a dimensão afeto positivo da escala BES com as dimensões ações e valores $(r=0,58)$ e autoconfiança $(r=0,23)$ da escala da resiliência. Dessa forma, o aumento da vivência de emoções positivas entre os diabéticos pode atuar de forma a incrementar melhor aceitação de si no tocante à doença e sua terapêutica.
\end{abstract}

Palavras-chave: diabetes mellitus; doença crônica; bem-estar subjetivo; resiliência psicológica.

\section{SUBJECTIVE WELL-BEING AND RESILIENCE IN PEOPLE WITH DIABETES MELLITUS}

\begin{abstract}
This article aims to measure the levels of Subjective Well-Being (SWB) and resilience in diabetics, as well as to verify the correlation between resilience and the factors that make up the subjective well-being. The SWB and resilience scales were applied in 104 diabetic patients (mean age $=56.16 ; S D=13.01$ ), living in the city of João Pessoa- PB The results showed that $65.4 \%$ of the participants presented high levels of SWB. With respect to resilience, it was verified that the majority have between moderate $(63.5 \%)$ and high capacity $(28.8 \%)$, besides a better performance in the dimension of actions and values. It was found that the positive affect dimension of the SWB scale correlated with the actions and values dimensions $(r=0.58)$ and self-confidence $(r=0.23)$ of the resilience scale. Thus, increasing the experience of positive emotions among diabetics, can act to increase better acceptance of self in relation to the disease and its treatment.
\end{abstract}

Keywords: diabetes mellitus; chronic disease; subjective well-being; resilience psychological. 


\title{
BIENESTAR SUBJETIVO Y RESILIENCIA EN PERSONAS CON DIABETES MELLITUS
}

\begin{abstract}
Resumen
El objetivo del siguiente artículo fue la medición de los niveles del Bienestar Subjetivo (BES) y la resiliencia en diabéticos, además de verificar la correlación entre la resiliencia y los factores que componen el bienestar subjetivo. Se aplicaron las escalas de BES y de resiliencia en 104 diabéticos (edad media $=56,16$, DP $=13,01$ ), residentes en la ciudad de João Pessoa - PB. Los resultados evidenciaron que el 65,4\% de los participantes presentaron altos niveles de BES. En cuanto a la resiliencia, se constató que la mayoría posee entre moderada $(63,5 \%)$ y alta capacidad $(28,8 \%)$, además de un mejor desempeño en la dimensión de acciones y valores. Se observó también correlación positiva entre la dimensión afectiva positiva de la escala BES con las dimensiones acciones y valores $(r=0,58)$ y autoconfianza $(r=0,23)$ de la escala de la resiliencia. De esta forma, el aumento de la vivencia de emociones positivas entre los diabéticos puede actuar para incrementar mejor aceptación de sí en lo referente a la enfermedad y su terapéutica.
\end{abstract}

Palabras claves: diabetes mellitus; enfermedad crónica; bienestar subjetivo; resiliencia psicológica.

\section{INTRODUÇÃO}

O presente artigo aborda o Bem-Estar Subjetivo (BES) e a resiliência no contexto do diabetes mellitus (DM), com a finalidade de fornecer maior visibilidade e compreensão de como vive esse grupo de pertença, no que tange à superação das limitações decorrentes dessa doença. Conforme Stuhler (2012) o diabetes pode ser ocasionado tanto por fatores genéticos quanto ambientais, os quais interferem no metabolismo das gorduras, das proteínas e da glicose, resultando no acúmulo desta última no organismo. Os tipos de diabetes mais frequentes são: o diabetes tipo 1 , que está relacionado a deficiência absoluta na secreção do hormônio insulina, resultante de uma destruição autoimune das células-beta do pâncreas; e o diabetes tipo 2, que é caracterizado pela ação inadequada de insulina (Rodríguez, Meneses, Toboso, \& Moreno, 2012).

Conforme a American Diabetes Association (ADA), o nível normal de glicose no sangue é de até $100 \mathrm{mg} / \mathrm{dL}$; acima deste, a pessoa pode apresentar crises hipo ou hiperglicêmicas (ADA, 2017). A doença inicia-se e progride de forma silenciosa a partir de um comprometimento disfuncional do pâncreas. Compreende um conjunto heterogêneo de doenças metabólicas associadas a complicações, disfunções e insuficiência de vários órgãos, que atingem paulatinamente os sistemas: oftalmológico, renal, neurológico e cardiovascular (Rodríguez et al., 2012).

Os principais sintomas do diabetes são: muita sede (polidipsia), excesso de urina (poliúria), muita fome (polifagia) e emagrecimento. Outros sintomas são: sonolência, dores generalizadas, formigamentos e dormências, cansaço doloroso nas pernas, câimbras, nervosismo, indisposição para o trabalho, desânimo, turvação da visão, cansaço físico e mental (Rodríguez et al., 2012).

Algumas pesquisas apontam que a incidência e a prevalência do diabetes tendem a aumentar em decorrência do envelhecimento populacional e da 
urbanização, mas, especialmente, devido ao estilo de vida atual, caracterizado por inatividade física e hábitos alimentares que predispõem ao acúmulo de gordura corporal (Flor \& Campos, 2017; Oliveira \& Vencio, 2014; Whiting, Guariguata, Weil, \& Shaw, 2011). De acordo com as estimativas mais recentes da Internacional Diabetes Federation (IDF), existem aproximadamente 415 milhões de pessoas no mundo com diabetes, elencando o rol das cinco doenças crônicas não transmissíveis mais frequentes. A perspectiva é que em 2040 haverá 642 milhões de pessoas diabéticas (IDF, 2015). No Brasil, cerca de 14,3 milhões de pessoas são portadoras da doença e aparecem 500 novos casos por dia, assim como se estima que $50 \%$ da população com DM tipo 2 desconheça seu diagnóstico, percebendo-o apenas após o aparecimento de suas complicações (Ministério da Saúde, 2014). Portanto, fazem-se necessárias algumas medidas profiláticas em relação ao estilo de vida dos diabéticos, que envolvam a reeducação alimentar, ingestão de medicamentos e de insulina e prática de atividades físicas, com vistas a reduzir a incidência das complicações decorrentes da doença (Otero, Zanetti, \& Ogrizio, 2008).

Este artigo fundamenta-se nos construtos teóricos do Bem-estar Subjetivo (BES) e da Resiliência. O BES consiste na avaliação subjetiva da qualidade de vida e compreende dois componentes fundamentais: o cognitivo e o afetivo. Este construto também é conhecido por felicidade, satisfação, estado de espírito e afeto positivo (Lima \& Morais, 2016; Snyder \& Lopez, 2009).

O componente cognitivo agrega os aspectos racionais e intelectuais, como julgamentos que o indivíduo faz sobre sua própria vida. Nessa perspectiva, podese considerar que a dimensão satisfação com a vida é entendida como um julgamento cognitivo, ou seja, um processo de juízo e de autoavaliação, que utiliza como parâmetros a comparação entre as circunstâncias de sua vida e o padrão definido por ela (Yilmaz \& Arslan, 2013).

O componente afetivo diz respeito aos sentimentos agradáveis e desagradáveis resultantes de vivências imediatas, denominados de afetos positivos e negativos, respectivamente (Siqueira \& Padovam, 2008; Diener, 1984). Nos afetos positivos, encontram-se as emoções prazerosas como o contentamento, interesse e entusiasmo, enquanto os afetos negativos se referem aos sentimentos ou emoções desagradáveis tais como: tristeza, ansiedade e medo (Snyder \& Lopez, 2009).

Desse modo, Diener e Lucas (2000) afirmam que o BES é mensurado positivamente, quando a pessoa se refere a altos níveis de satisfação com sua vida, alto níveis de emoções positivas e baixos níveis de emoções negativas. Sendo assim, esse construto representa uma avaliação pessoal sobre quão feliz o indivíduo se sente, independentemente do contexto e condições socioeconômicas, saúde, sucesso e outras variáveis que poderiam permitir uma avaliação objetiva de qualidade de vida (Oliveira, Nunes, Legal, \& Noronha, 2016; Nunes, Hutz, \& Giacomoni, 2009). 
Quanto ao construto da resiliência, este é definido como um processo dinâmico enquanto capacidade da pessoa de resistir e superar as adversidades, considerando os fatores intrínsecos e extrínsecos a ela (Pereira, Dutra-Thomé, \& Koller, 2016). Polleto e Koller (2008) assinalam que a resiliência está relacionada ao reforço das potencialidades do indivíduo, com a finalidade de resgatar e fortalecer as dimensões saudáveis das pessoas, fornecendo, assim, condições para a superação em situações de exposição de riscos. De acordo com Carvalho, Teodoro e Borges (2014) a capacidade resiliente é também processual e conquistada nas experiências da vida, considerada uma habilidade dinâmica, a qual é modificada dependendo do momento e das circunstâncias, e que impulsionando as pessoas para lidarem com a adversidade.

No contexto do diabetes, a resiliência seria a capacidade da pessoa em lidar com a patologia, aceitando suas limitações, colaborando com a aderência ao tratamento e readaptando-se a novos estilos de vida (Costa, 2017). Desse modo, a resiliência está relacionada aos processos psicossociais que influenciam no desenvolvimento saudável dos indivíduos, mesmo quando estes se encontram frente a situações problemáticas. Nesse eixo, o estudo da resiliência possibilita compreender quais características individuais e ambientais podem ser modificadas ou estimuladas para que as pessoas apresentem estratégias eficazes de enfrentamento da doença (Bianchini \& Dell'aglio, 2006; Silva et al., 2006).

O escopo de diferentes conceitos para a resiliência vai desde conceituações reducionistas com o foco exclusivamente na recuperação do trauma, até definições mais amplas que compreendem a resiliência como uma capacidade de proteção em curso que permite não só a recuperação reativa, mas também a aprendizagem pró-ativa, como forma de crescimento decorrente dos desafios enfrentados (Robertson \& Cooper, 2013). As pessoas podem ter trajetórias semelhantes, mas desenvolverem capacidades resilientes diferentes o que leva algumas a superarem as crises e outras não (Yunes, 2003).

A resiliência é determinada por fatores de proteção e de risco (Polleto \& Koller, 2008; Rutter, 1985). Os fatores de proteção, também denominados de buffers, são as influências que modificam, melhoram ou alteram a resposta de uma pessoa a algum perigo que predispõe a um resultado não adaptativo e que parecem mudar ou reverter circunstâncias potencialmente negativas, conhecidos como a autoestima, o apoio social, a aproximação interpessoal, a satisfação com a vida, o bem-estar subjetivo, a crença ou a religião etc. (Pesce, Assis, Santos, \& Oliveira, 2004; Rutter, 1985).

Os fatores de risco relacionam-se com eventos negativos os quais quando vivenciados aumentam a probabilidade da pessoa apresentar problemas biopsicossociais (Polleto \& Koller, 2008). Dessa maneira, os fatores de risco podem ser coadjuvantes ou incrementadores na probabilidade de os indivíduos sofrerem perturbações associadas aos eventos negativos de vida, os quais potencializam resultados disfuncionais de ordem física, social e/ou emocional 
(Pesce et al., 2004). Entre os fatores de risco estariam os eventos estressantes da vida, por exemplo, as perdas afetivas, experiências de doenças no próprio indivíduo ou na família, o desemprego, as guerras, as calamidades, a violência, entre outros (Rutter, 1985). Além disso, Pesce et al. (2004) consideram risco todo evento que se configure como empecilho ao nível individual ou ambiental e que potencialize a vulnerabilidade do indivíduo a resultados negativos.

No que tange à mensuração da resiliência, observa-se tanto no contexto nacional quanto internacional, a evidência de altos níveis de resiliência em pessoas com DM. No contexto nacional, destacam-se os trabalhos desenvolvidos por Tavares, Barreto, Lodetti, Silva e Lessmann (2011) e Böell, Silva e Hegadoren (2016) realizados em Florianópolis e Rosa e Mota (2016) em Minas Gerais. Segundo esses autores, o alto nível de resiliência pode estar relacionado à superação da ocorrência de eventos negativos, a exemplo do prognóstico do diabetes, impulsionando-os a novos estilos de vida.

No contexto internacional, citam-se os estudos de Jaser e White (2011) e Yi-Frazier et al. (2010), os quais consideraram que a resiliência nos diabéticos estaria associada a qualidade de vida, melhor controle metabólico, autoestima, autoeficácia, autodomínio, otimismo e esperança. Destaca-se também o estudo desenvolvido por Denisco (2011) em uma amostra de 71 mulheres afroamericanas com diabetes tipo 2, com o objetivo de verificar a relação entre os escores de resiliência e a hemoglobina glicolisada (HbA1c). Nesse estudo, observou-se que mais da metade dos participantes $(66,4 \%)$ foram classificados com alta resiliência, assim como constatou correlação positiva entre a resiliência e a HbA1c.

Pesquisas realizadas para avaliar a correlação entre os dois construtos em diferentes contextos apontam que quanto maior o nível de resiliência, melhor o BES (Zhao, Guo, Suhonen, \& Leino-Kilpi, 2016; Resende et al., 2010). Não obstante, no contexto do diabetes, são necessárias mais pesquisas, uma vez que essa relação tem sido pouco explorada. Nesse sentido, é objetivo deste estudo mensurar os níveis de bem-estar subjetivo e de resiliência em diabéticos, além de verificar a correlação entre a resiliência e os fatores que compõem o bemestar subjetivo (afetos positivos, negativos e satisfação com a vida).

\section{MÉTODO}

Trata-se de uma pesquisa de campo descritiva, de caráter transversal e de cunho quantitativo.

\section{Participantes}

Participaram do estudo 104 pessoas, com idades entre 19 e 79 anos ( $M=$ 56,16; $\mathrm{DP}=13,01)$, residentes no município de João Pessoa, PB, selecionados por uma amostragem não probabilística. Os participantes que responderam aos 
instrumentos nas clínicas foram selecionados por conveniência e, para os demais, se utilizou a amostragem por snowball ou "bola de neve", que consiste na localização inicial de algumas pessoas com o perfil necessário para a pesquisa, esses primeiros participantes são denominados de sementes. Nesse caso, as sementes auxiliam o pesquisador a iniciar seus contatos e a selecionar o grupo a ser estudado. Na sequência, solicita-se que as pessoas indicadas pelas sementes sugiram novos contatos com as características desejadas e assim sucessivamente (Vinuto, 2014). Adotou-se como critérios de inclusão: (i) ter idade igual ou superior a 18 anos e (ii) ter diagnóstico de DM.

\section{Instrumentos}

\section{Questionário Biossociodemográfico}

O Questionário foi utilizado com a finalidade de coletar informações acerca do perfil dos participantes, tais como: idade, sexo, escolaridade, estado civil, religião e renda. Além disso, questionou-se acerca do tipo de diabetes, tempo de diagnóstico e presença de complicações.

\section{Escala de Bem-Estar Subjetivo (EBES)}

Essa escala foi desenvolvida por Albuquerque e Tróccoli (2004) e é composta por duas subescalas de resposta tipo Likert de cinco pontos. A primeira é constituída por 47 itens, divididos em dois fatores: fator I (afetos positivos), totalizando 21 itens que responde a $24,3 \%$ da variância e alpha de Cronbach de 0,95; e o fator II (afetos negativos), que contabiliza 26 itens, responsável por $24,9 \%$ da variância e seu alfa é de 0,95. A segunda subescala corresponde ao fator III, denominada de satisfação com a vida versus insatisfação com a vida, composta por 15 itens correspondendo a 21,9\% da variância e alfa de 0,90 (Albuquerque \& Tróccoli, 2004).

Nesse instrumento, foram observadas correlações razoavelmente elevadas entre os fatores I e II $(r=0,36)$; I e III $(r=0,53)$; II e III $(r=0,47)$, o que sugeriu a presença de um fator geral. A análise fatorial mostrou que o fator geral, posteriormente denominado BES, é composto pelos 62 itens que explicam $32,9 \%$ da variância e apresenta um índice de fidedignidade $(\alpha)$ de 0,86 (Albuquerque \& Tróccoli, 2004).

\section{Escala de Resiliência}

A escala de Resiliência foi desenvolvida por Wagnild e Young (1993) e adaptada para o português numa amostra de escolares por Pesce et al. (2005) tendo sido constatado alpha de Cronbach $(\alpha)$ de 0,80 . Esse instrumento possui 25 itens descritos de forma positiva com as respostas do tipo Likert variando de 1 (discordo totalmente) a 7 (concordo totalmente), em que seu somatório total oscila de 25 a 175 pontos (Pesce et al., 2005). 


\section{Procedimentos e Considerações éticas}

A pesquisa foi aprovada pelo Comitê de Ética em Pesquisa da Universidade Federal da Paraíba - CEP/CCS, sob o protocolo de no 914.880, ano de 2014 e CAAE no 33087214.6.0000.5188. Durante a sua realização, foram respeitadas todas as condições éticas estabelecidas pela Comissão do Conselho Nacional de Saúde, criada pela Resolução no 466/2012.

Quanto ao procedimento de coleta dos dados, os diabéticos foram convidados a participarem da pesquisa em clínicas enquanto aguardavam a consulta médica, ou em outros locais, a exemplo da residência dos diabéticos. Inicialmente, cada participante assinou o Termo de Consentimento Livre e Esclarecido, bem como foram informados do caráter voluntário e do sigilo referente à sua identificação e, na sequência, procedeu-se a administração dos instrumentos, na seguinte ordem: questionário biossociodemográfico, escalas de BES e resiliência. O tempo total de aplicação dos instrumentos foi, em média, de 40 minutos.

\section{Análise dos dados}

Para a análise dos dados, utilizou-se o Pacote Estatístico para as Ciências Sociais (SPSS - 23.0), sendo realizadas estatísticas descritivas e inferenciais. A primeira foi empregada para descrever a média, desvio padrão e frequências do perfil da amostra e mensurar o BES e a resiliência.

A estatística inferencial foi utilizada para correlacionar os resultados dos dois construtos. Para tanto, realizou-se o teste de normalidade KolmogorovSmirnov para os construtos Resiliência e BES, adotando que a $\mathrm{H}_{0}$ : a distribuição se ajusta à normalidade, e a $\mathrm{H}_{1}$ : rejeita a distribuição normal. De modo que, para a resiliência, observou-se os valores $(D(101)=0,39 ; p=0,99)$ e para BES os dados $(D(70)=1,22 ; p=0,09)$. Como observado, não foi possível rejeitar a $\mathrm{H}_{\mathrm{o}}$, ou seja, ao nível de $5 \%$ de significância, admite-se que a distribuição dos construtos apresentam boa aderência ao modelo da distribuição normal. Nesse direcionamento, utilizou-se o teste de $r$ pearson para verificar a relação entre os fatores que compõe as referidas escalas.

Ressalta-se que para mensurar os níveis de BES, inicialmente obteve-se o escore total de cada subescala por meio da soma das respostas dos três fatores (afetos positivos, negativos e satisfação com a vida), dividido pelo número total de itens correspondente a estes fatores. Dessa forma, para se constatar o BES alto, fez-se necessário que a soma dos escores de afetos positivos com os escores de satisfação com a vida, fosse maior do que os escores de afetos negativos. Por outro lado, para se constatar o BES baixo, foi preciso que a soma dos escores de afetos positivos com o escore de satisfação com a vida resultassem num valor menor que os escores de afetos negativos (Santo, 2015; Albuquerque \& Tróccoli, 2004; Diener, 1984). 
Para avaliar o construto da resiliência, se utilizou as indicações propostas por Denisco (2011). Os escores entre 147 e 175 indicam altos níveis de resiliência; 121 a 146 indicam níveis moderados e as contagens menores que 121 indicam baixos níveis de resiliência.

\section{RESULTADOS}

Os resultados do questionário biossociodemográfico dos participantes estão apresentados na Tabela 1 . Observou-se que a maioria é do sexo feminino $82,7 \%$; casados $56,7 \%$; analfabetos e com ensino fundamental $39,4 \%$; quanto à religião $57,7 \%$ afirmaram serem católicos; $41,3 \%$ inseridos no mercado de trabalho assumindo diversas profissões, a saber: professores, domésticas, comerciantes e técnicos de enfermagem; 64,4\% possuíam renda de até um salário mínimo. No que tange às variáveis biológicas, notou-se que o tempo de diagnóstico variou de 1 a 36 anos ( $M=10,8 ; D P=9,11) ; 58,7 \%$ tinham entre 1 e 10 anos de diagnóstico da doença e a maioria $(95,2 \%)$ era diabéticos do tipo 2 . Identificou-se ainda que $62,5 \%$ dos diabéticos afirmaram não utilizar a insulina e $64,4 \%$ relataram ter alguma complicação decorrente da doença, a exemplo de problemas de visão, cardíaco, circulação e pressão alta.

Tabela 1.

Frequência e Porcentagem (\%) das Variáveis Biossociodemográficas das Pessoas com Diabetes $(n=104)$

\begin{tabular}{ccc}
\hline Variáveis & Frequência & $\%$ \\
\hline Sexo & & \\
Masculino & 18 & 17,3 \\
Feminino & 86 & 82,7 \\
\hline Estado Civil & & 56,7 \\
Casado & 59 & 43,2 \\
Solteiro & 45 & 39,4 \\
Escolaridade & & 32,7 \\
Fundamental & 41 & 27,9 \\
Médio & 34 & 57,7 \\
Superior & 29 & 33,7 \\
Religião & & 8,6 \\
Católica & 60 & \\
Evangélica & 35 & 41,3 \\
Outras & 09 & 35,6 \\
\hline Ocupação & & 23,1 \\
\hline Profissões diversas & 43 & \\
Aposentados & 37 & 24 \\
Donas de casa & &
\end{tabular}




\begin{tabular}{ccc}
\hline Renda Familiar & & \\
Até 1 salário mínimo & 67 & 64,4 \\
De 2 a 4 salários mínimos & 25 & 24,0 \\
De 5 a 10 salários mínimos & 09 & 8,7 \\
Superior a 10 sal. mínimos & 03 & 2,9 \\
\hline Tipo de Diabetes & & 95,2 \\
Tipo 2 & 99 & 4,8 \\
Tipo 1 & 05 & \\
Uso de Insulina & & 37,5 \\
Não & 65 & 58,7 \\
Sim & 39 & 27,9 \\
De 1 a 10 anos & & 13,5 \\
\hline De 11 a 20 anos & 61 & 64,4 \\
Superior a 21 anos & 29 & 35,6 \\
\hline Complicação & 14 & \\
Presença & & 67 \\
Ausência & 37 & \\
\hline
\end{tabular}

Em relação aos resultados encontrados por meio da escala de bem-estar subjetivo, a pontuação foi considerada no intervalo de 1 a 5 . No escore geral, a média obtida foi de 3,56 ( $D P=0,57)$. Em relação às dimensões, os resultados apontaram que o maior escore médio obtido foi o do afeto positivo, 3,65 (DP = $0,65)$, seguidos pelo de satisfação com a vida, média de $3,35(D P=0,31)$ e pelo de afeto negativo $(M=2,49 ; D P=0,64)$. Ao quantificar o $B E S$, identificou-se que mais da metade dos participantes apresentaram alto nível de BES, uma vez que $(65,4 \%)$ obtiveram a soma dos escores de afetos positivos com os escores de satisfação com a vida maior do que os escores de afetos negativos. A confiabilidade calculada foi verificada pelo alfa de Cronbach $(a=0,85)$, considerada satisfatória.

De acordo com uma análise mais detalhada dos indicadores das dimensões do BES, pode-se constatar que, para a dimensão cognitiva, no que tange aos afetos positivos, se destacou os sentimentos "decidido", com um valor médio de 4,06 ( $\mathrm{DP}=0,91)$. Os itens "determinado" e "ativo" foram pontuados igualmente com médias de 4,04 e desvio padrão de 1,02. Quanto aos afetos negativos, observou-se que os sentimentos de "ansiedade" $(M=3,58 ; D P=1,14)$ e "preocupação" ( $M=3,40 ; D P=1,18)$ foram os de maior destaque. O afeto negativo menos apresentado pelos participantes foi o item "agressivo" com um valor médio de 1,73 e desvio padrão de 0,89 . Quanto à dimensão de satisfação com a vida, observou-se que a colocação mais evidenciada foi "gosto da minha vida" com média de 4,47 ( $D P=0,87)$. 
No que tange à escala de Resiliência, constatou-se uma pontuação mínima de 104 e máxima de 167 pontos, com média de 138,19 ( $D P=13,94)$. Observouse que a pontuação da resiliência contabilizada segundo o modelo proposto por Denisco (2011) foi distribuída em três níveis: $28,8 \%$ com alta capacidade de resiliência, 63,5\% moderada e $7,7 \%$ pouca resiliência.

Analisou-se, também, a resiliência no que tange às dimensões que compõem esse construto, distribuídas conforme a escala utilizada. Nesse sentido, observou-se que o fator "ações e valores" obteve maior escore médio $(81,9)$ $(\mathrm{DP}=8,5)$, comparado aos demais, "autoconfiança e capacidade de adaptação a situações" ( $M=28,95 ; D P=4,6)$ e "independência e determinação" $(M=27,3$; $\mathrm{DP}=4,7)$.

Foi realizado o teste $r$ de Pearson, com o intuito de verificar a correlação entre a resiliência e os fatores que compõem o bem-estar subjetivo. Os dados podem ser observados na Tabela 2 . Os valores acima da diagonal da Tabela 2 correspondem ao $p$-valor, obtido em cada comparação e os índices na parte de baixo da diagonal representam os valores de $r$.

Tabela 2.

Correlação entre os fatores das escalas de resiliência e BES

\begin{tabular}{|c|c|c|c|c|c|c|c|}
\hline \multicolumn{2}{|c|}{ Escala } & 1 & 2 & 3 & 4 & 5 & 6 \\
\hline \multirow{3}{*}{$\begin{array}{l}\text { Bem-Estar } \\
\text { Subjetivo }\end{array}$} & 1 & - & 0,61 & 0,30 & 0,001 & 0,24 & 0,02 \\
\hline & 2 & $-0,05$ & - & 0,95 & 0,08 & 0,40 & 0,20 \\
\hline & 3 & 0,10 & $-0,01$ & - & 0,12 & 0,06 & 0,93 \\
\hline \multirow{3}{*}{ Resiliência } & 4 & 0,55 & $-0,17$ & 0,15 & - & 0,001 & 0,005 \\
\hline & 5 & 0,12 & $-0,08$ & 0,18 & 0,41 & - & 0,001 \\
\hline & 6 & 0,22 & $-0,12$ & $-0,01$ & 0,27 & 0,37 & - \\
\hline
\end{tabular}

Nota. 1. Afetos Positivos; 2. Afetos Negativos; 3. Satisfação com a Vida; 4. Ações e Valores; 5. Independência e Determinação; 6. Autoconfiança e Capacidade de adaptação.

De acordo com a Tabela 2 visualizou-se que a dimensão afeto positivo da escala BES, correlacionou-se significativamente e de forma positiva com as dimensões ações e valores $(r=0,55 ; p<0,001)$ e autoconfiança $(r=0,22, p=$ $0,02)$ da escala da resiliência, quanto ao fator independência não foi observada correlação. 


\section{DISCUSSÃO}

Os resultados advindos da Escala de BES apontaram que 65,4\% dos participantes apresentaram alto nível de BES, esses achados provavelmente indicam que, apesar da doença, os diabéticos vivenciam com maior frequência e intensidade sentimentos agradáveis e emoções prazerosas, a exemplo de se perceberem como pessoas decididas, determinadas e ativas e, no geral, estão satisfeitas com suas vidas, o que demonstra que a pessoa pode ter alto BES independentemente de sua condição de saúde. Esse resultado corrobora com o estudo de Oliveira et al. (2016) que apontou que a avaliação do BES é pessoal e independe de condições socioeconômicas, saúde, sucesso, entre outros aspectos.

Não obstante, Nunes et al. (2009) relatam que o medo de episódios de hiper ou hipoglicemia, o receio de complicações principalmente as cardíacas e a não adesão ao tratamento, podem interferem na diminuição do BES dos diabéticos. Outros autores como, Holmes-Truscott, Browne, Pouwer, Speight, \& Cummins (2015), Lyrakos et al. (2014) e Naess, Eriksen, Midthjell e Tambs (2004) observaram que, ao comparar as pessoas com diabetes e sem relatos de doenças, os diabéticos apresentaram menores índices de BES.

No que tange à resiliência, observou-se que mais da metade dos participantes possui entre moderada $(63,5 \%)$ e alta capacidade $(28,8 \%)$ para enfrentar as adversidades frente às limitações decorrentes do diabetes e sua terapêutica. Nesse sentido, pode-se dizer que elas demonstraram boas habilidades psicológicas, flexibilidade em aceitar o apoio familiar e social no auxílio para encarar as situações estressantes com vistas a uma adaptação bemsucedida. Esses achados corroboram a literatura a qual aponta que as pessoas com diabetes apresentam alta prevalência para a capacidade de resiliência (Böell et al., 2016; Denisco, 2011). Tavares et al. (2011) acrescentam, ainda, que os diabéticos que vivenciaram o agravamento de sua condição crônica e necessitaram de atendimento em uma unidade de emergência mobilizaram maior resiliência para o enfrentamento desta condição.

Ao analisar as dimensões da escala de resiliência, verificou-se que a dimensão "ações e valores" obteve maior destaque. O que significa que, na amostra, a resiliência das pessoas com diabetes encontra-se mais respaldada na ideia da competência pessoal e de aceitação de si mesmo e da vida. Esses resultados estão em consonância com Pesce et al. (2005) quando estes afirmam que essa dimensão se refere a dar sentido à vida, como é o caso de ter amizades, da realização pessoal, estar satisfeito com a vida e dar um significado para a mesma. De modo análogo, Pereira et al. (2016) acrescentam que a promoção da resiliência também pode ser dada considerando os fatores intrínsecos e extrínsecos, a exemplo das características pessoais como otimismo, boa autoestima, altruísmo, habilidade para resolver problemas, condições familiares, vinculação com adultos significativos e rede de apoio social. De modo 
amplo, pode-se dizer que para esse grupo de diabéticos essas características configuram-se como fatores promotores da resiliência.

Ao relacionar o BES com a resiliência, verificou-se que o afeto positivo se correlacionou com as dimensões ações e valores, e autoconfiança da escala de resiliência. Nesse direcionamento, esses achados indicam que o nível de emoções positivas vivenciadas pelas pessoas com diabetes influencia no desenvolvimento de competências pessoais voltadas para a autoaceitação, estabelecimento de relações interpessoais e autoconfiança. Esses resultados se coadunam com o estudo de Cassarino-Perez e Dell'Aglio (2015) cujo resultado indicou que fatores de proteção como suporte social, vinculação afetiva e características pessoais de autoestima, otimismo e altruísmo, colaboram para o aparecimento de processos de resiliência.

A aceitação da nova condição de vida por parte dos diabéticos é de suma importância para a colaboração com o tratamento proposto. A maior ou menor capacidade do paciente em controlar o sintoma da doença, bem como a obtenção de resultados a curto e longo prazo tem impacto positivo no bem-estar subjetivo das pessoas (Howell, Kern, \& Lyubomirsky, 2007). Figueiras (2013) observou que sessões psicoeducativas desenvolvidas com pacientes diabéticos podem contribuir para que estes adquiram competências favoráveis para o aumento dos afetos positivos e da satisfação com a vida, configurando melhor aceitação das condições de limitação decorrentes da doença. O bem-estar subjetivo está relacionado com melhoras nos resultados em saúde e na diminuição de comorbidades, embora o seu efeito ainda esteja em discussão (Howell et al., 2007). Conforme Vinaccia e Quiceno (2011) a resiliência é um dos fatores para explicar o paradoxo do bem-estar, que ocorre quando as pessoas doentes e com limitações no funcionamento cotidiano informam, mesmo assim, um bom nível de bem-estar.

Não obstante, o fato de o indivíduo apresentar altos níveis de BES e de resiliência não o deixa imune as comorbidades que decorrem do diabetes, uma vez que existe o fator genético da doença, que na maioria das vezes progride independente do controle do diabético, atrelado a esse aspecto se apresenta o não cumprimento da terapêutica proposta a qual inclui a ingestão de medicamentos, atividade física e reeducação alimentar, além da instabilidade emocional, como fora visualizado no estudo de Costa e Coutinho (2016). Destaca-se que os dados encontrados neste estudo não permitem inferir que a resiliência e o BES sejam preditores de saúde, embora, se saiba que as pessoas resilientes são mais otimistas, possuem mais energia e são mais flexíveis a adaptar-se a novas situações. Nesse direcionamento, Howell et al. (2007) alega que o BES está relacionado com melhoras nos resultados em saúde seja ela mental, física ou psicológica e na diminuição de comorbidades, embora o seu efeito ainda esteja em discussão. 


\section{CONSIDERAÇÕES FINAIS}

Este artigo teve como objetivo mensurar os níveis de bem-estar subjetivo e de resiliência em diabéticos, além de verificar a correlação entre a resiliência e os fatores que compõem o bem-estar subjetivo. Nesse direcionamento, os resultados apontaram altos níveis de BES e resiliência entre os diabéticos. Além disso, pôde-se verificar que as emoções positivas influenciam no desenvolvimento de competências pessoais voltadas para a autoaceitação, estabelecimento de relações interpessoais e autoconfiança, indicando a importância do fortalecimento desses fatores na vida das pessoas com diabetes.

Por fim, é necessário relatar algumas das limitações deste estudo, como é o caso da não generalização dos resultados, devido à utilização de uma amostra não probabilística, bem como, a quantidade de participantes. Destaca-se, também, as escalas aplicadas, uma vez que na literatura não existem instrumentos específicos para avaliar o BES e a resiliência em diabéticos, embora as escalas utilizadas já tenham sido empregadas em outros grupos de pessoas com DM. Como desdobramentos futuros, sugerem-se estudos prospectivos que avaliem o BES e a resiliência e os correlacionem ao diabetes; com isso poderia contribuir para verificar mecanismos de resiliência e BES ao longo do tempo associados aos fatores protetivos das comorbidades emocionais.

\section{DECLARAÇÃO DE CONFLITO DE INTERESSES}

Não há conflito de interesses.

\section{REFERÊNCIAS}

Albuquerque, A. S., \& Tróccoli, B. T. (2004). Desenvolvimento de uma escala de bem-estar subjetivo. Psicologia: Teoria e Pesquisa, 20(2), 153-164. doi: $10.1590 /$ S0102-37722004000200008

American Diabetes Association [ADA]. (2017). Diabetes mellitus. Recuperado de http://www.diabetes.org/are-you-at-risk/prediabetes/?loc=superfooter

Bianchini, D. C. S, \& Dell'Aglio, D. D. (2006). Processos de resiliência no contexto de hospitalização: Um estudo de caso. Paidéia, 16(35), 427-36. doi: 10.1590/S0103-863X2006000300013

Böell, J. E. W, Silva, D. M. G. V., \& Hegadoren, K. M. (2016). Sociodemographic factors and health conditions associated with the resilience of people with chronic diseases: A cross sectional study. Latin American Journal of Nursing, 24, 1-9. doi:10.1590/1518-8345.1205.2786

Carvalho, V. D., Teodoro, M. L. M., \& Borges, L. O. (2014). Escala de Resiliência para Adultos: Aplicação entre servidores públicos. Avaliação Psicológica, $13(2), 287-295$. 
Cassarino-Perez, L., \& Dell'Aglio, D. D. (2015). Processos de resiliência em adolescentes com diabetes Melittus tipo I. Psicologia em Estudo, 20(1), 4556. doi: 10.4025/psicolestud.v20i1.24035

Costa, F. G. (2017). Bem-estar subjetivo, resiliência e representações sociais no contexto do diabetes mellitus. (Tese de Doutorado). Universidade Federal da Paraíba, João Pessoa.

Costa, F. G., \& Coutinho, M. P. L. (2016). Representações sociais no contexto do Diabetes Mellitus. Psicologia em Estudo, 21(1), 175-185. doi: 10.4025/psicolestud.v21i1.29792

Denisco, S. (2011). Exploring the relationship between resilience and diabetes outcomes in African Americans. Journal of the American Academy of Nurse Practitioners, 23, 602-610. doi:10.1111/j.1745-7599.2011.00648.x

Diener, E. (1984). Subjective well-being. Psychological Bulletin, 95, 542-575.

Diener, E., \& Lucas, R. E. (2000). Explaining differences in societal level of happiness: Relative standards, need fulfillment, culture, and evaluation theory. Journal of Happiness Studies, 1(1), 41-78. doi: 10.1023/A:1010076127199

Figueiras, A. R. (2013). Bem-estar subjetivo e qualidade de vida em adultos com nefropatia diabética: impacto de uma intervenção psicológica. (Dissertação de Mestrado). Universidade do Algarve, Portugal.

Flor, L. S., \& Campos, M. R. (2017). Prevalência de diabetes mellitus e fatores associados na população adulta brasileira: Evidências de um inquérito de base populacional. Revista Brasileira de Epidemiologia, 20(1), 16-29. doi:10.1590/1980-5497201700010002

Holmes-Truscott, E., Browne, J. L., Pouwer, F., Speight, J., \& Cummins, R. A. (2016). Subjective well-being among adults with diabetes: Results from Diabetes MILES-Australia. Journal of Happiness Studies, 17(3), 1205-1217. doi:10.1007/s10902-015-9638-4

Howell, R. T., Kern, M. L., \& Lyubomirsky, S. (2007). Health benefits: Metaanalytically determining the impact of well-being on objective health outcomes. Health Psychology Review, 1, 83-136. doi: $10.1080 / 17437190701492486$

Jaser, S. S., \& White, L. E. (2011). Coping and resilience in adolescents with type 1 diabetes. Child: Care, Health and Development, 37(3), 335-342. doi: $10.1111 / j .1365-2214.2010 .01184 . x$

Lima, R. F. F., \& Morais, N. A. D. (2016). Caracterização qualitativa do bem-estar subjetivo de crianças e adolescentes em situação de rua. Temas em Psicologia, 24(1), 01-15. doi:10.9788/TP2016.1-01 
Lyrakos, G., Damigos, D., Chatziaggelaki, E., Papazafiropoulou, A. K., Koutsovasilis, A., Batistaki, C., ... Spinaris, V. (2014). Regression model for subjective wellbeing in patients with diabetes mellitus. The Journal of the European Psychiatric Association. 29(1), 1-9. doi:10.1016/S09249338(14)78749-8

Ministério da Saúde (2014). Estratégias para o cuidado da pessoa com doença crônica: diabetes mellitus. Brasília (DF). Recuperado de http://bvsms.saude.gov.br/bvs/publicacoes/estrategias_cuidado_pessoa_do enca_cronica_cab35.pdf

Naess, S., Eriksen, J., Midthjell, K., \& Tambs, K. (2004). Subjective well-being before and after the onset of diabetes mellitus: Results of the NordTrondelag Health Study. Journal of Diabetes and its Complications, 19(2), 88-95. doi:10.1016/j.jdiacomp.2004.05.003

Nunes, C. H. S., Hutz, C. S., \& Giacomoni, C. H. (2009). Associação entre bem estar subjetivo e personalidade no modelo dos cinco grandes fatores. Avaliação Psicológica, 8(1), 99-108

Oliveira, C. D., Nunes, M. F. O., Legal, E. J., \& Noronha, A. P. P. (2016). BemEstar Subjetivo: Estudo de correlação com as Forças de Caráter. Avaliação Psicológica, 15(2), 177-185. doi:10.15689/ap.2016.1502.06

Oliveira, J. E. P., \& Vencio, S. (2014). Diretrizes da Sociedade Brasileira de Diabetes: 2013-2014/Sociedade Brasileira de Diabetes. São Paulo, SP: AC Farmacêutica.

Otero, L. M, Zanetti, M. L., \& Ogrizio, M. D. (2008). Conhecimento do paciente diabético acerca de sua doença, antes e depois da implementação de um programa de educação em diabetes. Revista Latino-Americana de Enfermagem, 16(2), 231-237.

Pereira, A. S., Dutra-Thomé, L., \& Koller, S. H. (2016). Habilidades sociais e fatores de risco e proteção na adultez emergente. Psico, 47(4), 268-278.

Pesce, R. P., Assis, S. G., Avanci, J. Q., Santos, N. C., Malaquias, J. V., \& Carvalhaes, R. (2005). Adaptação transcultural, confiabilidade e validade da escala de resiliência. Cadernos de Saúde Pública, 17(4), 887- 896. doi: 10.1590/S0102-311X2005000200010

Pesce, R. P., Assis, S. G., Santos, N., \& Oliveira, R. D. (2004). Risco e proteção: Em busca de um equilíbrio promotor de resiliência. Psicologia: Teoria e Pesquisa, 20(2), 135-143.

Poleto, M., Koller, S. H. (2008). Contextos ecológicos: Promotores de resiliência, risco e proteção. Estudos de Psicologia, 25(3), 405-416. doi: 10.1590/S0103-166X2008000300009

Polleto, M., \& Koller, S. H. (2008). Contextos ecológicos: promotores de resiliência, fatores de risco e de proteção. Estudos de Psicologia, 25(3), 405-416. doi:10.1590/S0103-166X2008000300009 
Resende, M. C., Ferreira, A. A., Naves, G. G., Arantes, F. M. S., Roldão, D. F. M., Sousa, K. G., ... Abreu, S. A. M. (2010). Envelhecer atuando: Bem-estar subjetivo, apoio social e resiliência em participantes de grupo de teatro.

Fractal: Revista de Psicologia, 22(3), 591-608. doi:10.1590/S198402922010000900010

Robertson, I., \& Cooper, C. L. (2013). Resilience. Stress and Health, 29(3), 175176. doi: $10.1002 / \mathrm{smi} .2512$

Rodríguez, S. A., Meneses, A. L., Toboso, R. P. Q., \& Moreno, I. H. (2012). Diabetes mellitus. Medicine, 11(17), 995-1002.

Rosa, J. H. S., \& Motta, B. F. B. (2016). Aspectos sociais da resiliência em pacientes com diabetes mellitus tipo II. Revista Científica da Faculdade Governador Ozanam Coelho-Saúde, 1(1), 27-36.

Rutter, M. (1985). Resilience in the face of adversity: Protective factors and resistance to psychiatric disorder. British Journal of Psychiatry, 147, 598611. doi:10.1192/bjp.147.6.598

Santo, G. Q. L. (2015). Estratégias de coping e bem-estar subjetivo em trabalhadores de organizações de ensino superior. (Dissertação de Mestrado). Universidade Federal da Bahia, Salvador.

Silva, D. G. V., Francioni, F. F., Souza, S. S., Mattosinho, M. M. S., Coelho, M. S., \& Sandoval, R. C. B., ... Ferreira, N. (2006). Pessoas com Diabetes Mellitus: Suas escolhas de cuidados e tratamentos. Revista Brasileira Enfermagem, 59(3), 297-302. doi:10.1590/S0034-71672006000300009

Siqueira, M. M. M., \& Padovam, V. A. R. (2008). Bases teóricas de bem-estar subjetivo, bem-estar psicológico e bem-estar no trabalho. Psicologia: Teoria e Pesquisa, 24(2), 201-209. doi:10.1590/S0102-37722008000200010

Snyder, C. R., \& Lopez, S. J. (2009). Psicologia Positiva: Uma abordagem científica e prática das qualidades humanas. Porto Alegre, RS: Artmed.

Sociedade Brasileira de Diabetes. (2015). Diretrizes da Sociedade Brasileira de Diabetes. (3 ed). Itapevi, SP: A. Araújo Silva Farmacêutica.

Stuhler, G. D. (2012). Representações sociais e adesão ao tratamento do diabetes mellitus tipo 2. (Tese de Doutorado). Universidade Federal de Santa Catarina. Florianópolis, Santa Catarina.

Tavares, B. R. C., Barreto, F. D. A., Lodetti, M. L. L., \& Lessmann, J. C. (2011). Resiliência de pessoas com diabetes mellitus. Texto and Contexto Enfermagem, 20(4), 751-757. doi:10.1590/S0104-07072011000400014

Vinaccia, S., \& Quiceno, J. M. (2011). Calidad de vida relacionada conlasalud y factores psicológicos: Um estudio desde la enfermedad pulmonar obstructiva crónica-EPOC. Terapia Psicológica, 29(1), 65-75. doi: $10.4067 /$ S0718-48082011000100007

Vinuto, J. (2014). A amostragem em bola de neve na pesquisa qualitativa: Um debate em aberto. Temáticas, 22(44), 203-220. 
Wagnild, G., \& Young, H. (1993). Development and psychometric. Journal of nursing measurement, 1(2), 165-178.

Whiting, D. R. et al. (2011). IDF Diabetes Atlas: Global estimates of the prevalence of diabetes for 2011 and 2030. Diabetes Research and Clinical Practice, 94(3), 311-321.

Yi-Frazier, J. P., Smith, R. E., Vitaliano, P. P., Yi, J. C., Mai, S., Hillman, M., \& Weinger, K. (2010). A person-focused analysis of resilience resources and coping in patients with diabetes. Stress and Health, 26, 51-60. doi: $10.1002 /$ smi. 1258

Yilmaz, H., \& Arslan, C. (2013). Subjetive well-being, positive and negative affect in Turkish university students. The Online Journal of Counseling and Education, 2(2), 1-8. doi:10.1016/j.sbspro.2012.09.543

Yunes, M. A. M. (2003). Psicologia positiva e resiliência: O foco no indivíduo e na família. Psicologia em Estudo, 8, 75-84. doi:10.1590/S141373722003000300010

Zhao, F., Guo, Y., Suhonen, R., \& Leino-Kilpi, H. (2016). Subjective well-being and its association with peer caring and resilience among nursing vs medical students: A questionnaire study. Nurse Education Today, 37, 108-113. doi: $10.1016 /$ j.nedt.2015.11.019

\section{Sobre os autores}

Maria da Penha de Lima Coutinho é Doutora em Psicologia Clínica pela Universidade de São Paulo, pós-doutora pela Universidade Aberta de Lisboa, Portugal. Docente e coordenadora do curso de Psicologia do IESP, coordenadora do Núcleo de Pesquisa Aspectos Psicossociais de Prevenção e da Saúde Coletiva da UFPB. E-mail: mplcoutinho@gmail.com

Fabrycianne Gonçalves Costa é neuropsicóloga, mestre e doutora em Psicologia Social pela Universidade Federal da Paraíba, membro do Núcleo de Pesquisa Aspectos Psicossociais de Prevenção e da Saúde Coletiva da UFPB. Docente no curso de Psicologia do IESP. E-mail: fabrycianne@gmail.com Márcio de Lima Coutinho é doutor em Psicologia Social pela Universidade Federal da Paraíba, membro do Núcleo de Pesquisa Aspectos Psicossociais de Prevenção e da Saúde Coletiva da UFPB. Docente no curso de Psicologia do IESP. E-mail: coutinholmarcio@gmail.com

A contribuição de cada autor pode ser atribuída como se segue: F.G.C e M.P.L.C. contribuíram para a conceitualização, investigação, visualização e redação inicial do artigo (rascunho); F.G.C e M.L.C. foram responsáveis pelas análises estatísticas. Os três autores foram responsáveis pela redação final (revisão e edição).

Recebido em: 10/07/2017

$1^{\text {a }}$ revisão em: 02/11/2017

Aceito em: 30/05/2018 